\title{
An Aetiopathological Study on Epistaxis in Adults and its Management
}

Ajay Manickam, ${ }^{1}$ Debangshu Ghosh, ${ }^{1}$ Jayanta Saha, ${ }^{1}$ Sumit Kumar Basu ${ }^{1}$

\section{Introduction}

Epistaxis is a common clinical problem in ENT practice. Idiopathic aetiology is said to be the most common.

Aim of study

To find out whether idiopathic epistaxis is the most common cause of primary adulthood epistaxis and to formulate the best treatment protocol

Materials \& Methods

A prospective study was conducted in a tertiary healthcare setup from August 2013 to August 2014. 100 patients of more than 16 years of age of both sexes were studied. Patients presenting with active nasal bleeding in ENT ER and OPD were included. $\underline{\text { Results }}$

Truly idiopathic epistaxis was encountered in $37 \%$ of the study population followed by $20 \%$ cases in association with grossly deviated septum with septal spur(s). Most of the cases were managed by resuscitation alone with or without anterior nasal packing. Conclusion: With advances in endoscopy \& imaging techniques, cause of the epistaxis can most often be elicited and by sticking to a management protocol recurrence can be reduced. pistaxis is one of the commonest entities we come across in our day to day practice. History says it's a 2500 year old problem and each period of history describes a different aetiology for this problem. The term 'Epistaxis' was coined by William Cullen. Until the period of middle age, nasal bleeding was considered to be a natural means of purification of internal diseases. Hippocrates described a method to stop anterior nasal bleeding just by pinching the nose. He also stated that anterior plugging will stop bleeding from nose. By the first century BC, Scribonus Largus discovered the method of introducing rubber tube into the nose to stop bleeding and also secure the airway in the process. Egyptians used a special type of lichen grown in mummies "MUMIA" that had a haemostatic property for anterior nasal

1 - Department of ENT, RG Kar Medical College, Kolkata

Corresponding author:

Dr Debangshu Ghosh

email: ghoshdr.d777@ymail.com plugging. Morgagni predicted that endoscopy will play a major role in epistaxis management by 1761 .

\section{Relevant anatomy}

The nasal cavity is supplied by both the internal and the external carotid artery systems. The sphenopalatine artery and the greater palatine artery supply the posteroinferior part of the nasal cavity while the anterior and posterior ethmoidal arteries supply its superior part. Branches of the facial artery supply the anteroinferior part of the nasal cavity. The common sites of nasal bleeding are: (1) Kiesselbach plexus in the Little's area is the most common site of anterior nasal bleeding. (2)Woodruff's plexus, the venous plexus situated at the posterior end of the inferior turbinate, is often a major contributor to posterior epistaxis.

\section{Classification of Epistaxis ${ }^{1}$}

Epistaxis can be primary (no proven causal factor) or 
secondary (proven causal factor), childhood ( $<16$ years) or adulthood ( $>16$ years), anterior (bleeding point anterior to pyriform aperture) or posterior (bleeding point posterior to pyriform aperture). Various aetiologies that have been put forward for epistaxis are weather (chronobiology), NSAIDs, alcohol, hypertension and septal deformity. Seventy to eighty percent of adulthood epistaxis is considered to be idiopathic where no proven causal factor can be determined.

\section{Aims and Objectives}

1. To identify whether primary epistaxis is the most common cause of adulthood epistaxis

2. To formulate the best management protocol in a tertiary healthcare setup for management of epistaxis in adults and prevention of its recurrence

\section{Materials and Methods}

A prospective study was conducted in the Emergency Department and the Department of ENT in our institution. Patients included in the study were those aged more than 16 years presenting with epistaxis without a known causal factor. The study period was from August 2013 to August 2014. The sample size was one hundred. Patients presenting with history of trauma, children below sixteen years of age and patients with a known proven cause of epistaxis (secondary epistaxis) were excluded from the study.

The patients, after signing a written informed consent, were subjected to detailed history taking and thorough clinical examination to come to a diagnosis. Patients were managed with or without nasal packing depending upon the situation. Vital signs were closely monitored. After resuscitation, proper clinical evaluation was done for detection of the cause of the nosebleed. The required investigations like complete haemogram and routine examination of urine, diagnostic nasal endoscopy and radiological investigations were carried out subsequently as indicated. In cases of epistaxis where a sinonasal tumour was subsequently detected, punch biopsy was done for histopathological confirmation. Special tests were done for systemic diseases, if indicated. Surgical management like endoscopic sinus surgery and open surgery were done according to the pathology. Management of epistaxis was done in a stepwise manner, starting from nose pinching, cauterization of visible bleeding points, anterior with or without posterior nasal packing and sphenopalatine artery ligation, if necessary (Fig.1). Although uncommon, it is essential to exclude systemic disorders as a cause of unexplained recurrent epistaxis.

\section{Observations}

74 of the patients in this series were males and 26 were females. Male to female ratio was 3:1. Incidence of nose bleeding was maximum in persons over 40 years of age (Fig. 2). Most of the incidents occurred between the months of November and February (Fig. 3). Out of 100 patients, 46 were hypertensive and 54 were normotensive. Moreover, among the 46 hypertensive patients, 18 were in the idiopathic group, 19 were on nonsteroidal antiinflammatory drugs (NSAID) and 9 patients had gross septal deviation (DNS) with septal spur (Fig. 4). Among 54 normotensive patients, 19 had idiopathic epistaxis, 24 had a definite secondary cause of epistaxis and 11 patients had gross septal deviation (Fig. 5).

Five patients had Juvenile nasopharyngeal angiofibroma. CECT scan of nasopharynx was done in those patients. All the patients were above the age of 16 years and less than 20 years of age. All went for surgical resection of the tumour either externally or under endoscopic guidance. One such patient had another bout of epistaxis and was diagnosed to have a recurrent growth. Transpalatal excision was done in that patient. Three patients were diagnosed to have sinonasal polyposis. Plain and CECT scan of paranasal sinuses were done, followed by endoscopic sinus surgery. Two patients had septal ulcers that were diagnosed by endoscopy and was treated by chemical cauterisation. 


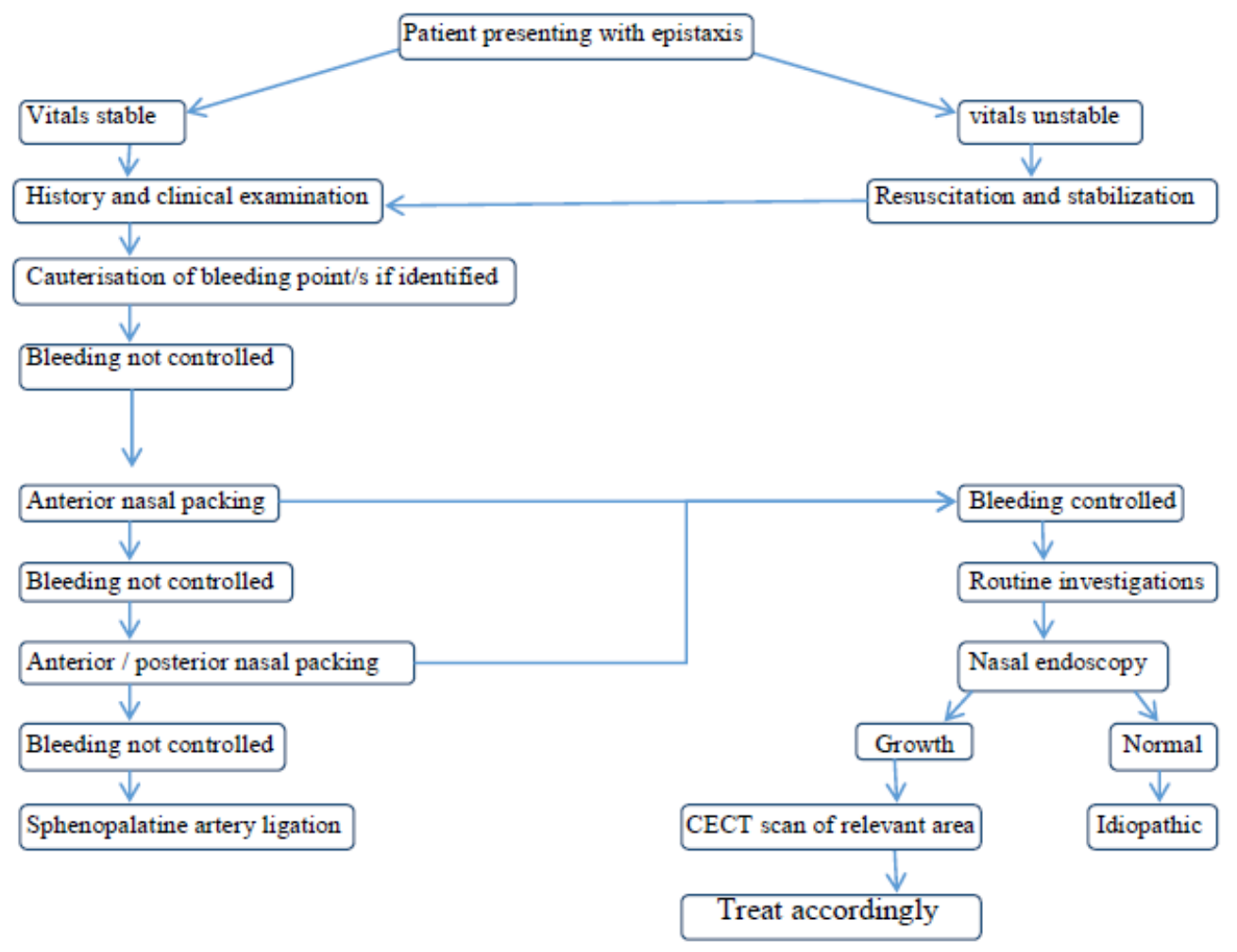

Fig. 1 Management algorithm of epistaxis in our setup

Two patients had rhinosporidiosis. Endoscopy guided excision and cauterization of the base was done. Table I shows various aetiologies of epistaxis from the group of patients who were provisionally grouped as idiopathic epistaxis. Table II outlines management options of such patients.

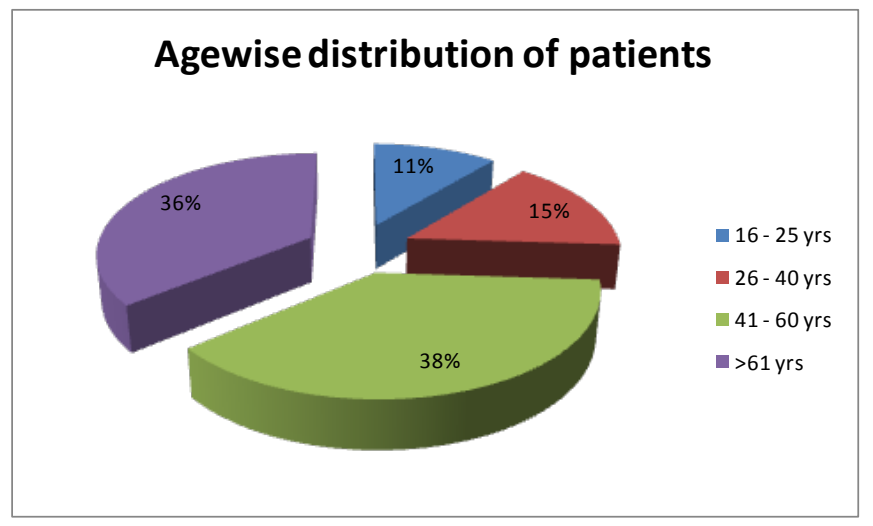

Fig. 2 Distribution of patients according to age

\section{Discussion}

Adult primary epistaxis is a variant that challenges ENT surgeons the most. A total of 100 patients having nasal bleeding without a known causal factor above the age of 16 years were included in our study. The present study had $76 \%$ males and $24 \%$ females. Males outnumbered females in our study. Petruson B et al. studied the frequency of epistaxis in an adult population sample and described that epistaxis was a more common presentation in the male population. ${ }^{2}$ In our study, we got a similar finding. The incidence of epistaxis was more in the winter months from November to February (67\%). Watkinson JC et al. in their study found similar seasonal incidence of epistaxis. ${ }^{3}$ Nunez DA et al. studied relationship of weather with nose bleeding and opined that admissions were greatest in months of winter. ${ }^{4}$ Thus, there is a strong relationship between 


\section{Epistaxis: Chronobiology}

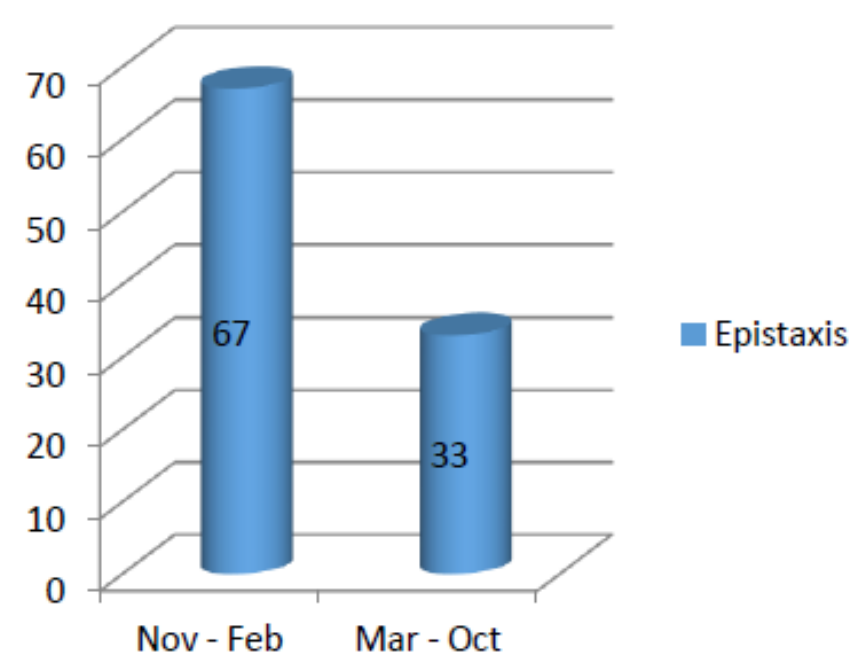

Fig. 3 Chronobiology of Epistaxis

epistaxis and winter probably due to drying effect of nasal mucous membrane from exposure to cold air. Thus, chronobiology and its relation with epistaxis were proved.

\section{Normotensive epistaxis}

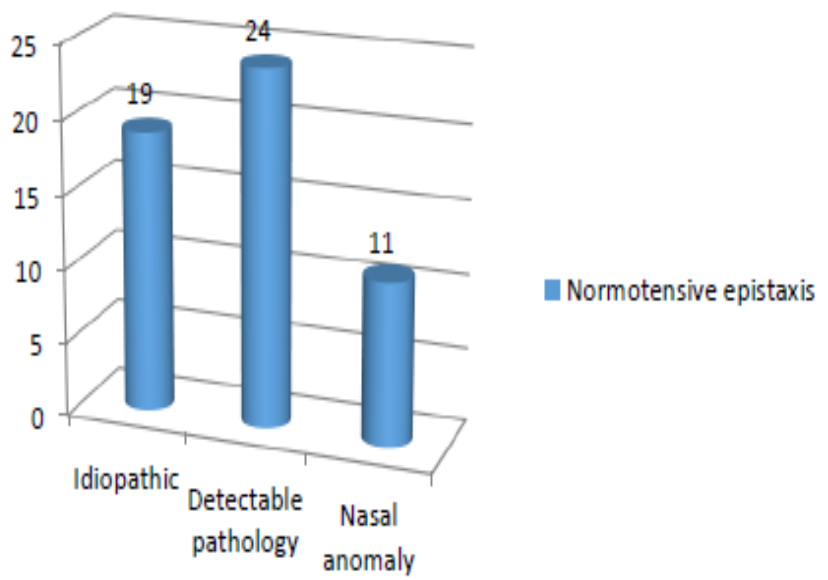

Fig. 5 Epistaxis in normotensive patients and its causes

\section{Epistaxis in}

\section{hypertensive patients}

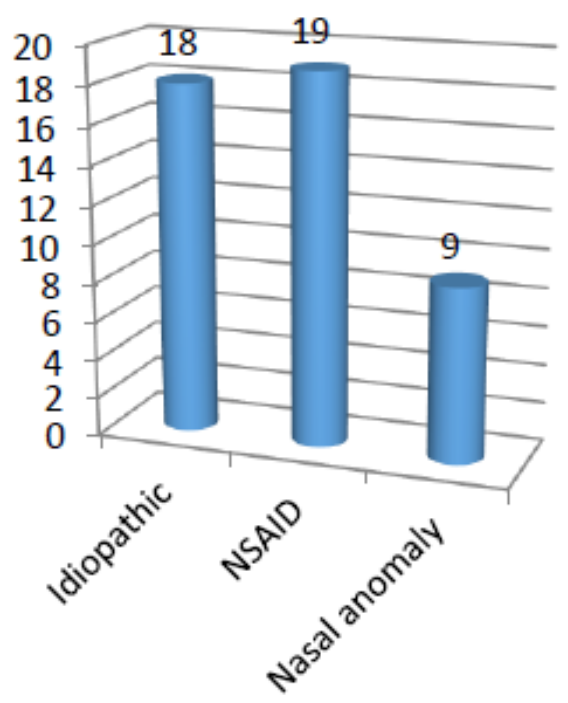

Epistaxis

Fig. 4 Epistaxis in hypertensive patients and its causes

Most of the patients were above the age of 40 years. According to the available medical literature, the commonest cause of adulthood epistaxis is idiopathic, which accounts for 70 to $80 \%$ of the cases. Mcgarry GW described the most common cause of adulthood epistaxis as idiopathic. ${ }^{1}$ In our study, the most common cause of epistaxis was found to be secondary epistaxis. Idiopathic epistaxis accounted for only $37 \%$ of the cases. Among 100 patients, 46 were diagnosed to be hypertensive and the rest 54 were normotensive. Ecevit MC et al. in their study at university of Dokuzz showed that $56.7 \%$ epistaxis patients had elevated blood pressure. In our study, we found nearly $46 \%$ were having elevated blood pressure. Although hypertension should always be considered as an associated finding in epistaxis, it should never be taken as the sole aetiology. ${ }^{5}$ Lubianca et al. in their study proved the association between hypertension and epistaxis to be inconclusive. ${ }^{6}$ This apparent elevation of blood pressure may be a result of anxiety associated with hospital admissions. ${ }^{1}$

Among hypertensive patients, 5 patients had 
recurrent history of epistaxis. Among normotensive patients, 4 patients had recurrence. Lammens et al. in their study showed that $1 / 3$ rd of the patients attending ENT emergency were on anticoagulant therapy. ${ }^{7}$ In our study, 19 (1/5th.) patients were taking anticoagulant medication for cardiological problems. Shergordoskey et al. described use of tricholoroacetic acid to stop anterior nasal bleeding. Chemical cautery of the bleeding point(s) detected by nasal endoscopy provide almost $90 \%$ control of symptoms. ${ }^{8}$ Two patients with nasal septal ulcers, in this series, were treated with chemical cauterization and epistaxis was controlled effectively without any recurrence. Thus, endoscopy guided chemical cautery can also be described as an important management option to control mucosal bleeding points.

Table I: Aetiology of Epistaxis in study population

\begin{tabular}{|cc}
\hline AETIOLOGY OF EPISTAXIS & PERCENTAGE \\
\hline $\begin{array}{c}\text { Idiopathic (cause could not be } \\
\text { determined) }\end{array}$ & 37 \\
\hline $\begin{array}{c}\text { Gross DNS with septal spur } \\
\text { NSAID }\end{array}$ & 20 \\
\hline Septal angioma & 19 \\
\hline Nasopharyngeal angiofibroma & 7 \\
\hline Sinonasal polyposis & 5 \\
\hline Septal ulcers & 3 \\
\hline Nasopharyngeal carcinoma & 2 \\
\hline Rhinosporidiosis & 2 \\
\hline Deranged liver function & 2 \\
\hline Thrombocytopenia & 1 \\
\hline
\end{tabular}

Liu $\mathrm{J}$ et al. did a retrospective study on epistaxis and described the importance of endoscopy in patients presenting with epistaxis.. ${ }^{9}$ O'Leary and Weymuller also advocated rigid endoscopy as a tool for control of nosebleeds and had nearly $90 \%$ success rate. ${ }^{10} \mathrm{We}$ managed most of our patients by resuscitation alone or with anterior nasal packing.

Two male patients were HIV positive and had abnormal liver function. Deranged liver function was the cause of epistaxis in these patients. Hoff BV et al. noted that liver plays a major role in the clotting mechanism in our body and deranged liver function can lead to nasal bleeding. ${ }^{11}$ It is important to rule out any liver function abnormality in idiopathic epistaxis.

Villock et al. in their study showed that endoscopy guided sphenopalatine artery cauterization in idiopathic recurrent epistaxis gave promising results and recurrence was minimal. It reduced the recurrence of epistaxis in nearly $98 \%$ patients. ${ }^{12}$ Three of the 54 normotensive patients had history of recurrent epistaxis which were

Table II: Management of Epistaxis in study population

\begin{tabular}{|cc|}
\hline MANAGEMENT & PERCENTAGE \\
\hline Resuscitation & $97 \%$ \\
\hline Anterior nasal packing & $48 \%$ \\
\hline $\begin{array}{c}\text { Anterior and posterior nasal } \\
\text { packing }\end{array}$ & $3 \%$ \\
\hline Nasal endoscope guided surgeries & $16 \%$ \\
\hline Open surgeries & $6 \%$ \\
\hline Other systemic diseases managed & $22 \%$ \\
\hline
\end{tabular}

idiopathic in nature. Endoscopy assisted sphenopalatine artery ligation was done. There was no recurrence. Hence, in patients with idiopathic refractory epistaxis, endoscopic sphenopalatine artery ligation or cauterisation is a very important option of management. O'Reilly $\mathrm{BJ}$ et al. studied the association between epistaxis and septal deviation in adults. They described that there was no significant association between septal deviation and epistaxis but the study was only an observational non-experimental one. ${ }^{13}$ Septal deviation or spur was associated with $20 \%$ of the patients of epistaxis in this study. Septoplasty was done in 6 patients. However, randomised controlled trials are necessary to establish 
the actual correlation between epistaxis and deviation of the nasal septum.

\section{Conclusion}

Epistaxis is one of the most common ENT emergencies and is associated with different aetiologies. Primary adulthood epistaxis is a challenge to ENT surgeons. Resuscitation and stabilisation of the patient is the first line of management. Diagnosis can be reliably made by endoscopy and radiological study. Contrary to the earlier belief that $70-80 \%$ of adult epistaxis is idiopathic, our study reveals that majority of cases of epistaxis not only have a definite cause but also have various options available for treatment. However, further multicentric population based studies are required to evaluate various aetiologies of epistaxis. With advances in science, use of nasal endoscopy and CT scan, ENT surgeons now have several options to look for the particular cause of epistaxis. Adherence to a strict protocol in the management of patients of epistaxis may lead us to definite aetiological diagnoses in a large section of patients. Thus, it is possible to avoid grouping most of the cases of epistaxis as idiopathic. Targeting the aetiological factors during treatment may help to further reduce the chances of recurrence.

\section{Acknowledgement}

The authors would like to thank the Principal, R.G.Kar Medical College, Kolkata for allowing them to conduct the research with clearance from the Institutional Ethical Committee.

\section{References}

1. McGarry GW. Epistaxis. In Michael Gleeson Ed. Scott Brown's otolaryngology 7th edition vol. 2. London: Hodder Arnold publication; 2008; 1603-1608

2. Petruson B, Rudin R. The frequency of epistaxis in a male population sample. Rhinology. 1975; 13:129-133

3. Watkinson JC et al. Epistaxis in: Mackay IS Bull TR Ed volume 18. London: Butterworth's, 1997; 5-7

4. Nunez DA, McClymont LG, Evans RA. Epistaxis the study of relationship with weather. Clinical Otolaryngology. 1998; 23:280

5. Ecevit MC, Erdag CK. ENT emergencies in a tertiary care setup. In their study at university of Dokuzz. Available at www. pubmed.com [cited on 07/01/2015]

6. Lubianca Neto JF, Fuschs FD, Facco SR, Gus M. Epistaxis: evidence of end organ damage in patients with hypertension? Clinical rhinology. 1992;19:111-115

7. Lammens Lemkken Laureyls et al. Emergencies in ENT. Available at www.pubmed.com [cited on 07/01/2015]

8. Shergordoskey et al. Role of chemical cautery in the management of anterior epistaxis. Journal of Rhinology. 1998; $57: 125$

9. Liu. J , Zhonga, Wang. Role of nasal endoscopy in the management of acute epistaxis. Journal of Otolaryngology. 1996; 11:153-158

10. O'LearyStickney, Weymuller EA. Rigid endoscopy for the control of epistaxis. Archives of Otolaryngology and Headneck surgery. 1992;118:966-7

11. Hoffbrand V, Moss P, Petit J. Essential haematology 5th edition Massachusetts: Blackwell publishing professional, 2006; 218

12. Villock \& Jones $\mathrm{K}$ et al. Comparative study in patients, who went on arterial ligation and embolization in patients who had recurrent history of epistaxis. Acta Medica Scandinavica Journal of Internal Medicine. 1988.

13. O'Reilly BJ, Simpson DC, Dharmaratenam R Septal deviation in adults and epistaxis.Clinical Otolaryngology. 1996; 21:12-4. 